\title{
SIMULAÇÃO DA OXIDAÇÃO DO TOLUENO UTILIZANDO LINGUAGEM VISUAL BASIC FOR APPLICATIONS
}

\author{
M. H. A. A. CRUZ ${ }^{1}$, H. L. SENZANO ${ }^{2}$, V. M. F. BEZERRA ${ }^{3}$, C. P. SOUZA ${ }^{4}$, \\ A. L. LOPES-MORYIAMA ${ }^{5}$
}

${ }^{1}$ Universidade Federal do Rio Grande do Norte, Departamento de Engenharia Química

${ }^{2}$ Universidade Federal do Rio Grande do Norte, Programa de Pós-Graduação em Engenharia Química

${ }^{3}$ Universidade Federal do Rio Grande do Norte, Departamento de Engenharia Química

${ }^{4}$ Universidade Federal do Rio Grande do Norte, Departamento de Engenharia Química

${ }^{5}$ Universidade Federal do Rio Grande do Norte, Departamento de Engenharia Química E-mail para contato: coffee_cruz@hotmail.com

\begin{abstract}
RESUMO - Este trabalho tem como objetivo explorar o Excel®, ferramenta de planilhas bastante conhecida e presente na maioria dos aparelhos do mundo, de modo a testar sua capacidade científica de simular modelos de reações catalíticas através de sua linguagem de programação VBA® (Visual Basic for Applications). Através dessa linguagem, foi simulado um modelo obtido na literatura que descreve a cinética de uma reação de oxidação catalítica do tolueno. Foi obtido um gráfico que relaciona a concentração de tolueno com a posição no reator de modo que os inputs, ou seja, as informações do sistema e da reação podem ser alteradas livremente de modo a permitir o estudo de diferentes dimensões de reator, vazões, concentrações iniciais, taxas de reação e parâmetros para o catalisador. Os resultados demonstram que é possível simular reações catalíticas com quantidades consideráveis de características variáveis valendo-se de uma ferramenta presente em nosso cotidiano.
\end{abstract}

\section{INTRODUÇÃO}

Atualmente faz-se necessário o acesso a plataformas que ofereçam segurança quando se trata de simulação de processos a partir de dados reais. Infelizmente, esse tipo de ferramenta se torna inacessível a muitos frente aos altos custos e até complexidade de manuseio. Outro tópico ainda moderno no mundo científico é o desenvolvimento de pesquisas acerca de reações catalíticas - oportunidade de tornar processos cada vez mais baratos e eficazes por meio da economia de recursos e exploração de mecanismos reacionais.

Tendo em vista tais demandas, este trabalho tem como objetivo explorar o potencial científico de softwares comuns tais como o Excel®, da Microsoft ${ }^{\circledR}$, por meio de simulações. Mais especificamente, trataremos da simulação da oxidação catalítica do tolueno, cujo modelo cinético está disponível na literatura.

Visual Basic for Applications ${ }^{\circledR}$ é o nome dado à linguagem de programação computacional criada pela Microsoft que se vale de uma maneira de trabalho gráfica e 
demonstrativa. A linguagem é um acrônimo para "código de instrução multifuncional para iniciantes" em inglês, e o termo "visual programming" se refere a qualquer linguagem que permite aos usuários a manipulação gráfica dos seus elementos.

Esta linguagem é tida como fácil de utilizar e prática em suas aplicações para variados fins. Além disso, está disponível em muitos softwares da Microsoft ${ }^{\circledR}$, tais como o Word®, Power Point ${ }^{\circledR}$ e o programa de planilhas Excel ${ }^{\circledR}$. Particularmente para este, o VBA ${ }^{\circledR}$ se torna uma ferramenta poderosa e acessível, principalmente em relação ao fornecimento e disposição de dados.

\section{REVISÃO BIBLIOGRÁFICA}

No universo atual de desenvolvimento de reações catalíticas tem-se como um dos principais objetivos a conversão fotocatalítica do metano. Nota-se nos trabalhos de TAYLOR (2003), por exemplo, que eles se valem do oxido de tungstênio como catalisador para obter metanol. Os tipos de reatores são em sua maioria anelares ou tubulares, sendo do primeiro tipo nos trabalhos de NOCETI et al. (1997),

O papel da luz nestas pesquisas é justamente de complementar os efeitos dos catalisadores uma vez que em alguns casos é necessária a formação de radicais para o acontecimento das reações, como é observado nos trabalhos de TAYLOR et al. (1997).

Existe uma série de propriedades que interferem no rendimento de um reator fotocatalítico. Elas influenciam em aspectos como velocidade e mecanismos da reação, equilíbrio químico e até a natureza dos produtos formados. Catalisadores são substâncias cuja finalidade é reduzir a energia de ativação de uma reação e por consequência alterar sua velocidade (lei de Arrhenius). Logo, sua concentração está diretamente relacionada com o efeito global que causam num processo. Isso pode ser observado na Figura 1, do trabalho de ASGARI et al. (2012).

Figura 1 - Eficiência de uma reação de extração de fenol vs concentração de catalisador

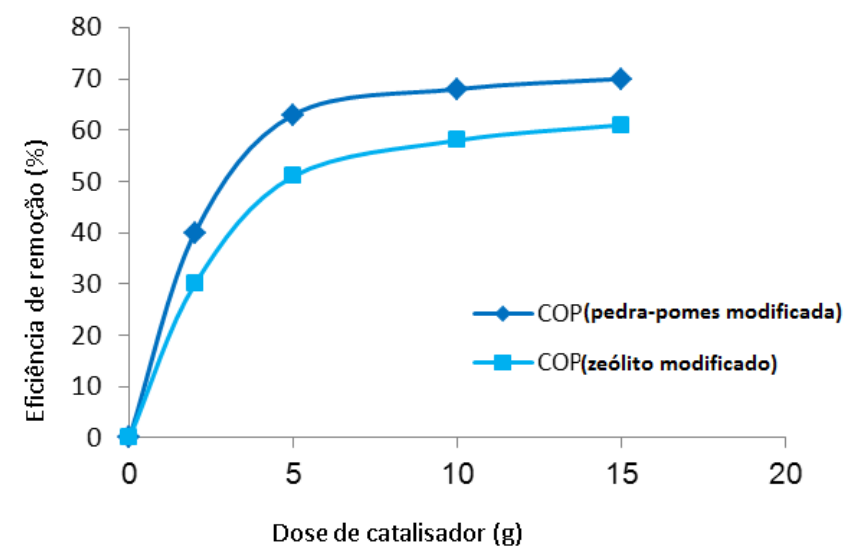


O equilíbrio químico e a cinética de uma reação são diretamente influenciados pelas concentrações das substâncias presentes no reator. Por isso, as vazões de entrada e saída, as extensões de reações paralelas e o regime de escoamento (com ou sem acúmulo) são características mensuráveis responsáveis pelas concentrações de cada componente.

Utilizou-se o trabalho de TOMASIC et al. (2008) de modelagem da oxidação fotocatalítica do tolueno em reator tubular. O "modelo 1", como foi chamado, é representado pela seguinte equação cinética:

$$
-u \frac{d C_{A}}{d z}=k_{g} a\left(C_{A, g}-C_{A, s}\right)
$$

Onde:

$$
\begin{aligned}
& k_{g}=\frac{S h D_{A}}{2 R(1-k)} \\
& S h=0,705\left(\operatorname{Re} \frac{R}{L}\right)^{0,43} S c^{0,56} \\
& S c=D_{g} \rho / \mu \\
& \operatorname{Re}=\frac{\left(2 R(1-k) u_{m} \rho\right)}{\eta}
\end{aligned}
$$

Onde:

$\mathrm{a}$ - área geométrica de superfície $\left(\mathrm{m}^{2}\right)$;

$\mathrm{C}_{\mathrm{A}}$ - concentração do composto " $\mathrm{A}$ " $\left(\mathrm{mol} \mathrm{m}^{-3}\right)$;

$\mathrm{C}_{\mathrm{A}, \mathrm{g}}$ - concentração de "A" na fase gasosa $\left(\mathrm{mol} \mathrm{m}^{-3}\right)$;

$\mathrm{C}_{\mathrm{A}, \mathrm{s}}$ - concentração de "A" no catalisador (sólido) $\left(\mathrm{mol} \mathrm{m}^{-3}\right)$;

$\mathrm{D}_{\mathrm{A}}$ - coeficiente de difusão molecular no $\operatorname{ar}\left(1 \mathrm{bar}, 20^{\circ} \mathrm{C}\right)\left(\mathrm{m}^{2} \mathrm{~s}^{-1}\right)$;

$\mathrm{D}_{\mathrm{g}}$ - coeficiente de difusão molecular $\left(\mathrm{m}^{2} \mathrm{~s}^{-1}\right)$;

$\eta$ - viscosidade dinâmica $\left(\mathrm{m}^{2} \mathrm{~s}^{-1}\right)$;

$\mathrm{k}$ - Constante de taxa da reação $\left(\mathrm{mol} \mathrm{m}^{-3} \mathrm{~s}^{-1}\right)$;

$\mathrm{k}_{\mathrm{g}}$ - coeficiente de transferência de massa na interface $\left(\mathrm{m} \mathrm{s}^{-1}\right)$;

$\mathrm{L}$ - comprimento do reator $(\mathrm{m})$;

$\mu$ - viscosidade cinemática $\left(\mathrm{mol} \mathrm{m}^{-1} \mathrm{~s}^{-1}\right)$;

$\rho$ - densidade $\left(\mathrm{mol} \mathrm{m}^{-3}\right)$;

$\mathrm{R}$ - raio do reator (m);

Re - número de Reynolds;

$\mathrm{u}$ - velocidade linear do fluido $\left(\mathrm{m} \mathrm{s}^{-1}\right) ; \mathrm{u}_{\mathrm{m}}$ - velocidade linear média do fluido $\left(\mathrm{m} \mathrm{s}^{-1}\right)$. 
As considerações para este modelo são que o estado é estacionário, isotérmico, pressão constante ao longo do reator, escoamento ideal, o catalisador encontra-se nas paredes internas do reator tubular em estado sólido, difusão interfásica desprezível e desativação catalítica desprezível. Este modelo não leva em consideração efeitos da luz. Além disso, tomamos a liberdade de considerar que a concentração de tolueno em fase sólida $\left(\mathrm{C}_{\mathrm{A}, \mathrm{s}}\right)$ é muito menor do que em fase gasosa $\left(\mathrm{C}_{\mathrm{A}, \mathrm{g}}\right)$.

Ressalta-se que foi adicionado um sinal negativo à Equação 1 pois ela é referente à taxa de variação da concentração do tolueno, que é um reagente.

\section{METODOLOGIA}

Para simular a concentração de tolueno ao longo do reator, a Equação 1 foi integrada e a seguinte função foi obtida:

$$
C_{A, g}(z)=C_{A, 0} / \exp \left[\frac{k_{g} a z}{u}\right]
$$

Onde $\mathrm{C}_{\mathrm{A}, 0}$ é a concentração inicial de tolueno.

Utilizando-se o software Excel® 2013, da Microsoft ${ }^{\circledR}$, através da linguagem VBA®, foi criada uma macro que gera dados de posição no reator e concentração de tolueno de acordo com informações de entrada disponíveis na planilha. Estas informações estão descritas na Tabela 1 .

Tabela 1 - Tabela de dados de entrada

\begin{tabular}{|l|l|}
\hline$u(m / s)$ & \\
\hline$C A, 0(m o l / m 3)$ & \\
\hline$R(m)$ & \\
\hline$L(m)$ & \\
\hline$D A(m 2 s-1)$ & \\
\hline$D g(m 2 s-1)$ & \\
\hline$k(m o l m-3 s-1)$ & \\
\hline$\rho(m o l m-3)$ & \\
\hline$\mu(m o l m-1 s-1)$ & \\
\hline$\eta(m 2 s-1)$ & \\
\hline$a(m 2)$ & \\
\hline
\end{tabular}

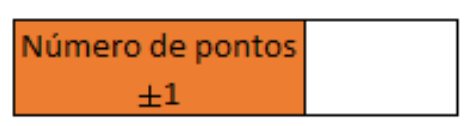

O código faz com que os dados sejam escritos numa das planilhas do programa, a qual produz um gráfico de concentração de tolueno em fase gasosa versus a posição no reator. 


\section{RESULTADOS}

Como resultado da simulação, tem-se uma ferramenta que consiste em duas planilhas (uma para entrada de dados e outra para saída) que fornecem informações de concentração de reagente tolueno ao longo do reator tubular. Como mostrado na Tabela 2, para uma entrada de informações quaisquer, é possível prever a concentração de tolueno através do gráfico da Figura 3.

Tabela 2 - Tabela de dados de entrada preenchida

\begin{tabular}{|c|c|}
\hline $\mathrm{u}(\mathrm{m} / \mathrm{s})$ & 0,05 \\
\hline $\mathrm{CA}, 0(\mathrm{~mol} / \mathrm{m} 3)$ & 5 \\
\hline $\mathrm{R}(\mathrm{m})$ & 1 \\
\hline $\mathrm{L}(\mathrm{m})$ & 10 \\
\hline DA $(\mathrm{m} 2 \mathrm{~s}-1)$ & 0,03 \\
\hline $\operatorname{Dg}(\mathrm{m} 2 \mathrm{~s}-1)$ & 0,03 \\
\hline$k(\mathrm{~mol} \mathrm{~m}-3 \mathrm{~s}-1)$ & 0,0004 \\
\hline$\rho(\mathrm{mol} \mathrm{m}-3)$ & 1 \\
\hline$\mu(\mathrm{mol} \mathrm{m}-1 \mathrm{~s}-1)$ & 1 \\
\hline$\eta(\mathrm{m} 2 \mathrm{~s}-1)$ & 1 \\
\hline$a(m 2)$ & 31,42 \\
\hline \begin{tabular}{|c} 
Número de pontos \\
\pm 1
\end{tabular} & 10 \\
\hline
\end{tabular}

Figura 3 - Conjunto de dados e gráfico resultante $\mathrm{C}_{\mathrm{A}, \mathrm{g}}\left(\mathrm{mol} / \mathrm{m}^{3}\right)$ versus $\mathrm{z}(\mathrm{m})$

\begin{tabular}{r|r|}
\hline CA,g & $z$ \\
\hline & \\
5,000 & 0 \\
4,396 & 1 \\
3,865 & 2 \\
3,398 & 3 \\
2,988 & 4 \\
2,627 & 5 \\
2,309 & 6 \\
2,030 & 7 \\
1,785 & 8 \\
1,569 & 9 \\
1,380 & 10
\end{tabular}
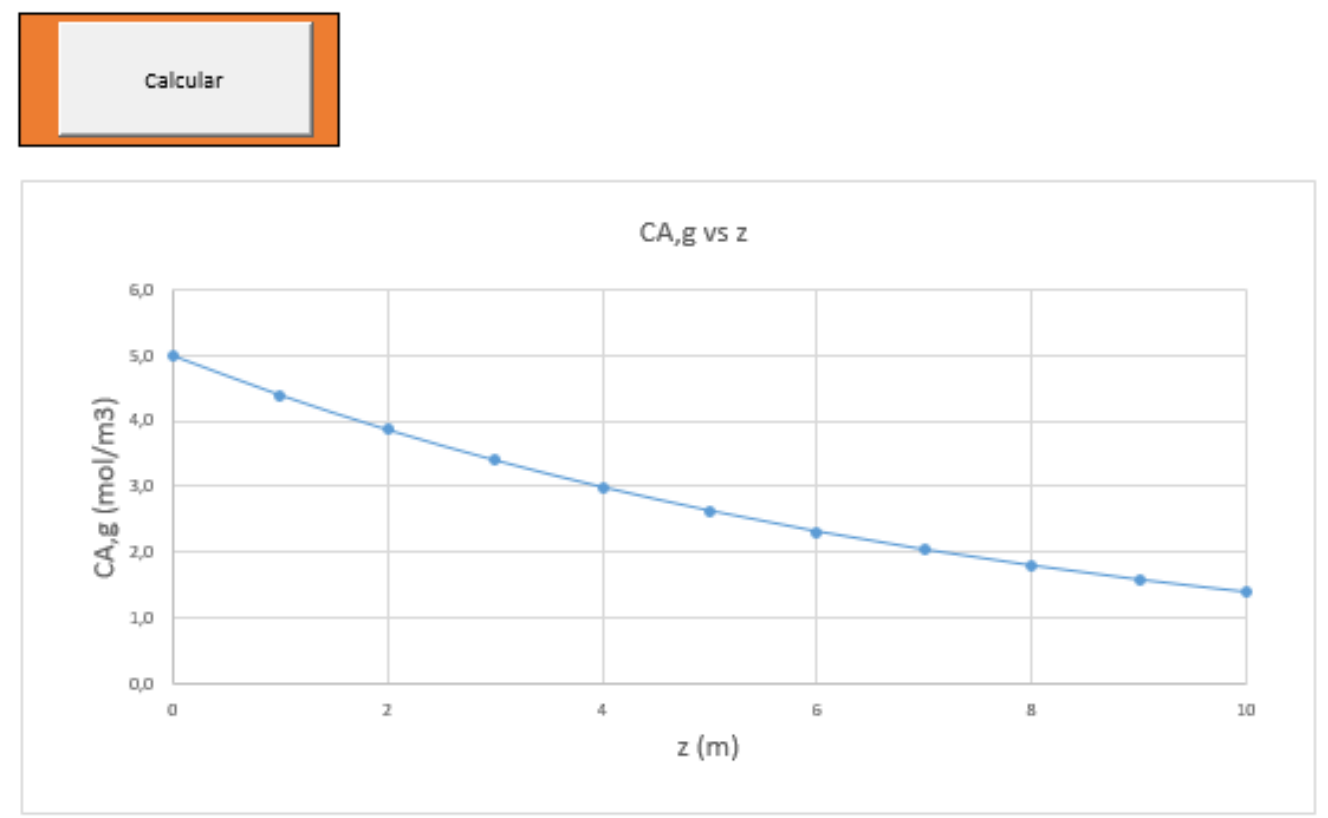


\section{CONSLUSÃO}

Observa-se, conforme a Figura 3, que o perfil de desaparecimento de $\mathrm{C}_{\mathrm{A}, \mathrm{g}}$ ao longo do reator está de acordo com o modelo desenvolvido da literatura.

O "modelo 1" desenvolvido por TOMASIC et al. (2008) não envolve o efeito da luz na reação nem ajustes relacionados à transferência de massa na interface. Porém, é fruto de dados experimentais, o que o torna uma aproximação válida bem como torna válida sua simulação através do VBA®. Além disso, reações catalíticas de ordem similar em reator tubular podem valer-se do código construído para simulações com viés prático.

\section{REFERÊNCIAS}

ASGARI, G; RAHMANI, A; ASKARI, F. B.; GODINI, K. Catalytic Ozonation of Phenol Using Copper Coated Pumice and Zeolite as Catalysts. Journal of Research in Health Science, v. 12, p. 93-97, 2012.

NOCETI, R. P.; TAYLOR, C. E.; D’ESTE, J. R.; Photocatalytic Conversion of Methane. Catalysis Today, v. 33, p. 199-204, 1997.

TAYLOR, C. E.; NOCETI, R. P.; D’ESTE, J. R.; Photocatalytic Conversion of Methane to Methanol. Pittsburg Energy Technology Center.

TAYLOR, C. E.; Methanol and Hydrogen From Methane, Water, and Light. Prepr. Pap.-Am. Chem. Soc., Div. Fuel Chem 48.2 (2003): 876.

TOMASIC, V.; JOVIC, F.; GOMZI, Z. Photocatalytic oxidation of toluene in the gas phase: modelling an anular photocatalytic reactor.Catalysis Today, v. 137, p. 350-356, 2008. 